\title{
Imaging Evaluation of Collaterals in the Brain: Physiology and Clinical Translation
}

\author{
Sunil A. Sheth • David S. Liebeskind
}

Published online: 27 November 2013

(c) Springer Science+Business Media New York 2013

\begin{abstract}
The cerebral collateral circulation is a network of blood vessels designed to preserve cerebral blood flow when primary routes fail. Though recognized for hundreds of years, the beneficial influence of collateral flow has now gained significant attention because of widely available, rapid, and real-time non-invasive imaging techniques. Multimodal CTand MRI-based techniques, with angiographic and perfusion assessments, are becoming mainstays in the care of patients with ischemic brain disease. These methods allow for precise delineation of the structural and functional aspects of cerebral blood flow and as such provide valuable information that can inform the diagnosis and treatment of cerebral ischemia in both the acute and chronic setting.
\end{abstract}

Keywords Stroke · Angiography · Perfusion · Intracranial stenosis - Thrombectomy $\cdot$ Moyamoya

\section{Introduction}

The cerebral collateral circulation is an evolutionarily conserved network of blood vessels designed to maintain consistent cerebral perfusion in the face of physiologic and pathophysiologic changes. Our recent ability to qualitatively and quantitatively define the structural and functional

This article is part of the Topical Collection on Advances in NeuroImaging.

S. A. Sheth · D. S. Liebeskind $(\square)$

UCLA Stroke Center, 710 Westwood Plaza, Los Angeles, CA 90095, USA

e-mail: davidliebeskind@yahoo.com

S. A. Sheth

e-mail: ssheth@post.harvard.edu aspects of this system through non-invasive imaging techniques has revolutionized our approach to cerebral ischemia. From a diagnostic point of view, these methods identify brain territories at risk and inform the likely clinical course of the patient, in terms of progression of infarct in the case of acute ischemia, or recurrent stroke in the case of chronic disease. From a therapeutic point of view, these data are invaluable in determining which patients present a favorable vascular profile with tissue that could be saved with revascularization. Further, because reperfusion procedures in acute and chronic ischemic brain disease are imprecise and in many cases unproven $[1 \bullet \bullet, 2,3 \bullet, 4]$, demonstrating the degree of restoration of blood flow and correlating with clinical outcome is crucial in the development of these techniques. Ultimately, collateral imaging provides rich details on the flow of blood to different regions of the brain; it is the characteristics of this flow, and not those of the arterial lesion, that determine whether the underlying brain parenchyma lives or dies.

\section{Collateral Circulation Anatomy}

The cerebral collateral circulation is a system of redundancies within the neurovasculature designed to preserve cerebral blood flow when primary routes fail [5]. It is the principle component of the brain's homeostatic response to ischemic insults. As a result of this network of subsidiary vessels that includes components of both the arterial and venous circulation, the brain is able to survive occlusions of even large proximal arteries; almost $60 \%$ of patients have been shown to tolerate complete occlusions of the internal carotid artery [6].

The anatomy of this circulation includes extracranial sources of blood flow that can be diverted to intracranial 
vessels as well as intracranial routes that can supplement other intracranial areas in need [5]. Extracranial carotid branches that can shunt flow via anastomoses to the intracranial arteries include the facial, maxillary, middle meningeal, and occipital arteries. Common anastomotic routes include the ophthalmic artery, which may fill in a retrograde direction, as well as smaller and unnamed dural arteries.

Intracranial collateral routes can be further subdivided into primary and secondary routes. The primary pathways include the components of the circle of Willis, and the secondary pathways include less direct routes that develop over time. The anterior portion of the circle of Willis includes the anterior communicating artery, which allows for interhemispheric collateralization and blood flow from the contralateral carotid artery. This collateral route would result in blood flow reversal in the ipsilateral proximal anterior cerebral artery. The posterior portion contains the posterior communicating arteries, which allow for collateralization from the posterior circulation to the anterior circulation, or vice versa. Variability and asymmetry is the rule in population studies of circle of Willis anatomy, with an intact circle present in a minority of patients [7]. Overall, the number and quality of collateralization is highest between anterior and middle cerebral arteries, with less robust connections between posterior cerebral and middle cerebral arteries [8]. Dynamic changes in these Willisian routes may be chronicled with serial imaging of stroke patients, and ongoing studies may provide further dimension to the functional impact of specific configurations in this proximal network.

Secondary intracranial pathways consist of pre-existing collateral routes that do not normally feed the territory in question, but in the setting of occlusion of the primary artery can assume additional responsibility to support the area at risk, as demonstrated in Fig. 1. These pathways take time to develop and as such may be of lessened utility in acute occlusions. Examples of such vessels include the ophthalmic artery and leptomeningeal branches, which have been shown to become more prominent in the setting of carotid occlusions. Other less commonly discovered collateral pathways include tectal plexus branches that connect the supratentorial posterior cerebral arteries with the infratentorial superior cerebellar artery. The rete mirabile caroticum, found in most lower vertebrates, can rarely be found in humans and connects the internal and external carotid circulations [9].

Beyond these inborn collateral vessels, humans are able to generate new vessels in areas of ischemia through angiogenesis and arteriogenesis [10]. Angiogenesis refers to the sprouting of new vessels from pre-existing ones [11]. Stimulated by parenchymal ischemia, vascular growth factors are released and bind to endothelial cells to signal proliferation, migration and eventually development of new mature vessels. Arteriogenesis consists of the maturation of pre-existing vessels in the setting of upstream occlusion. While this process shares some similarities with angiogenesis, arteriogenesis operates through different signaling mechanisms and is driven primarily by the shear stress of the pressure gradient between high and low perfusion zones. Arteriogenesis results in the creation of new arterioles independent of ischemic signaling through the development and growth of pre-existing capillaries that develop a smooth muscle layer and expand to withstand additional blood flow [12]. Both processes are slow and likely would only provide benefit in instances of chronic, as opposed to acute, ischemia. Observations of angiogenesis have been made in rodent models of middle cerebral artery occlusion [13] and may correlate with the finding in humans that a slower pace of cerebral ischemia is more likely to be tolerated [14]. These processes play a large role in the development of additional collaterals following surgical revascularization procedures, as shown in Fig. 2.

The robustness of the collateral network likely diminishes with age and other vascular comorbidities such as hypertension. The effect of aging was studied in middle cerebral artery occlusions in mice and was shown to lead to a proportional decrease in collateral vessels, with narrower diameter and increased tortuosity. Resistance of these vessels was increased, as was the ultimate infarct volume compared to younger animals [15]. Hypertension has also been shown to lead to less robust collaterals in rodent models, with narrower resultant anastomoses and decreased blood flow [13]. Ongoing studies are investigating such correlations with the human collateral circulation of the brain in a variety of stroke populations.

\section{Structural Collateral Imaging Defines Vascular Lesions}

The gold standard for the anatomic evaluation of the collateral circulation is digital subtraction angiography (DSA). This technique allows for dynamic visualizations of blood flow that cannot be attained through other traditional imaging modalities. It assesses all three major routes: extracranial to intracranial, through the circle of Willis, and through leptomeningeal vessels [16]. The primary limitation of DSA is its invasive nature, as well as its reliance on iodinated contrast and ionizing radiation. In addition, there may be variability in the appearance of the cerebral vasculature, particularly smaller distal vessels, depending on the volume and pressure of the contrast injection 5].

Non-invasive techniques have now become standard in the initial assessment of primary and collateral cerebral circulations. CT angiography (CTA) is one of the most widely available and utilized diagnostic tools in assessing arterial occlusions in the setting of acute ischemic stroke 

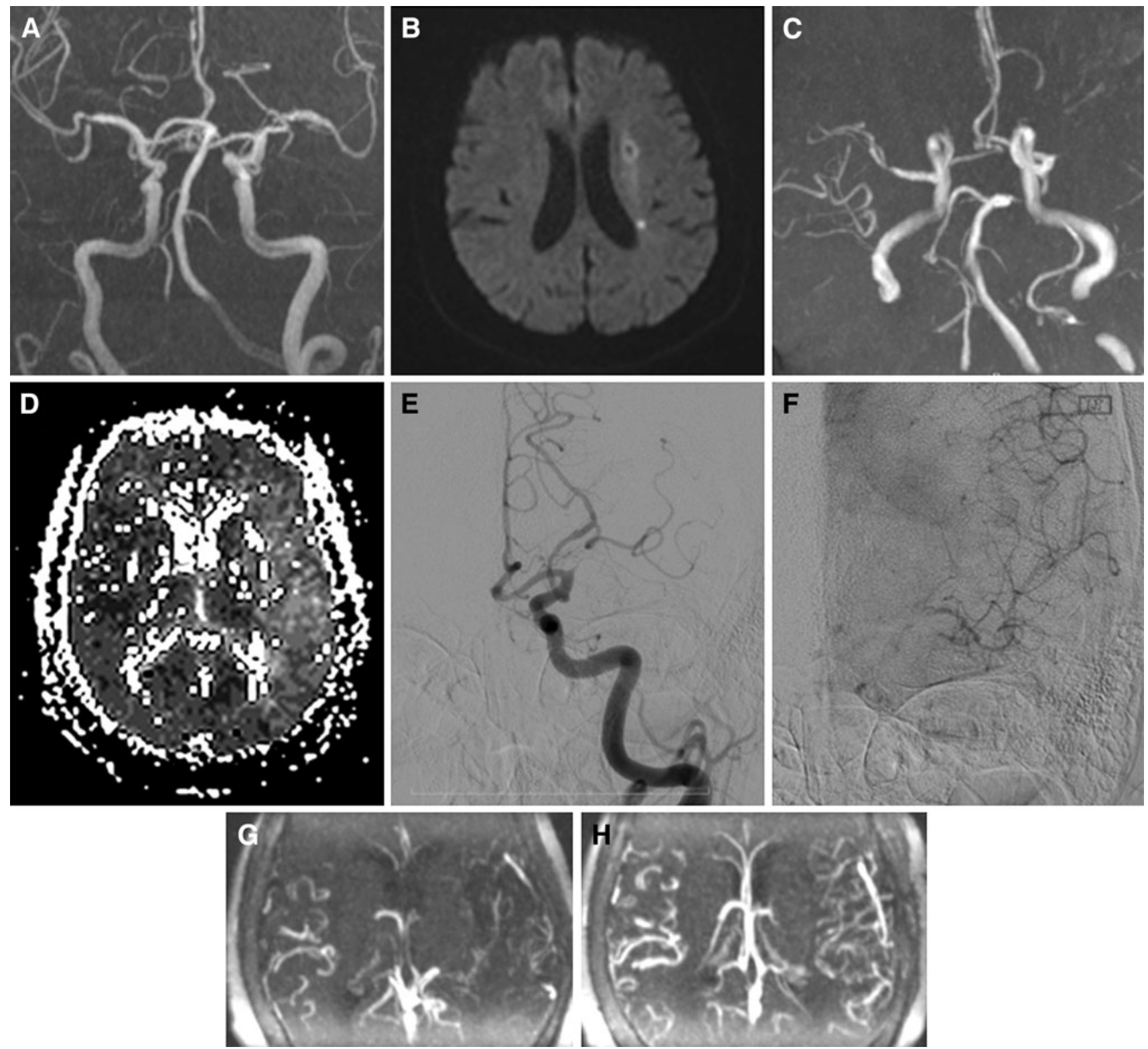

Fig. 1 A 65-year-old woman with a history of hypertension presented with recurrent paroxysmal episodes of right face and arm weakness associated with aphasia. a MR angiography (MRA) revealed stenosis of the bilateral middle cerebral arteries (MCAs), worse on the left than right. One year later in spite of medical management, her symptoms became more frequent, to near daily. b Diffusion-weighted imaging after one of these spells revealed acute on chronic ischemia, and $\mathbf{c}$ repeat MRA showed worsened left MCA stenosis. d MR perfusion revealed delayed time to peak in the

and can be used to concurrently determine the presence or absence of structural collaterals. CTA data can be interpreted from the source images, or reformatted as maximal intensity projections (MIPs) or multiplanar reconstructions (MPRs). Dynamic CTA, also known as 4D CTA, is a more recently developed and promising new technique that allows for dynamic assessments of cerebral blood flow that results in data comparable to cerebral angiography. These

majority of the left MCA distribution. e, f Conventional angiography revealed robust collateralization of the left MCA territory from the left posterior cerebral artery, which had led to the preservation of the majority of the MCA territory in spite of near occlusion. The patient underwent extracranial to intracranial bypass with improvement in vessel filling on MRA on $\mathbf{g}$ preoperative to $\mathbf{h}$ postoperative evaluations. The patient has remained symptom free 3 months after her surgery

data can been acquired through a 320-row CT scanner with dynamic acquisitions once per second or by reconstructions of data acquired utilizing CT perfusion acquisitions. This approach has demonstrated good correlation with cerebral angiography in detecting arteriovenous malformations [17]. In acute ischemic stroke, the additional time-delayed images allow for enhanced delineation of the extent of arterial occlusions [18]. 

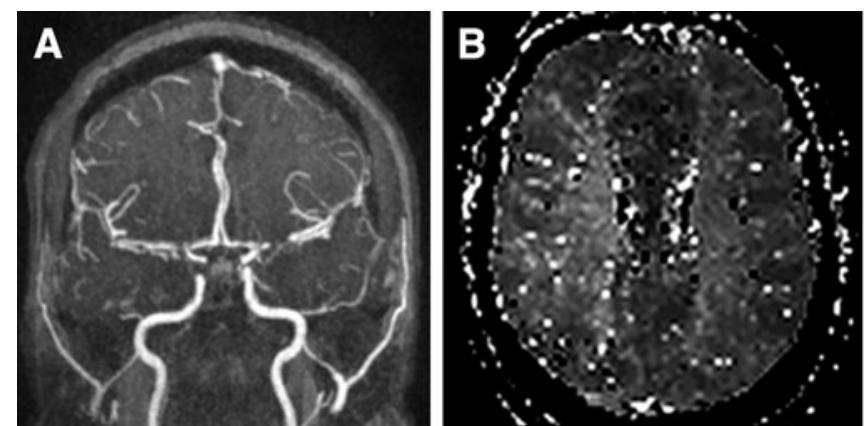

Fig. 2 A 29-year-old woman presented for evaluation of recurrent paroxysmal spells of left hand paresthesias as well as new onset headaches. a MR angiography (MRA) revealed stenoses of the bilateral internal carotid (ICA) and middle cerebral arteries (MCAs). b MR perfusion showed delayed time to peak in the territory of the
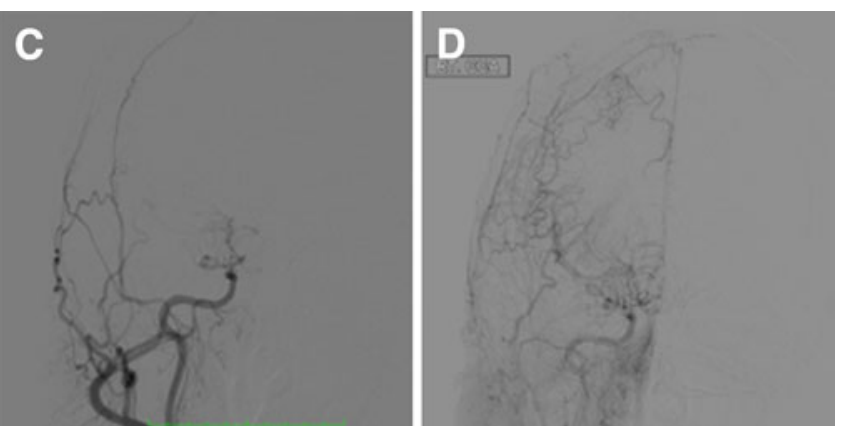

right MCA compared to the left. c Conventional angiography confirmed near occlusion of the right ICA with moyamoya like changes and $\mathbf{d}$ robust collateralization of the extra carotid through dural feeders after an encephaloduroarteriosynangiosis procedure
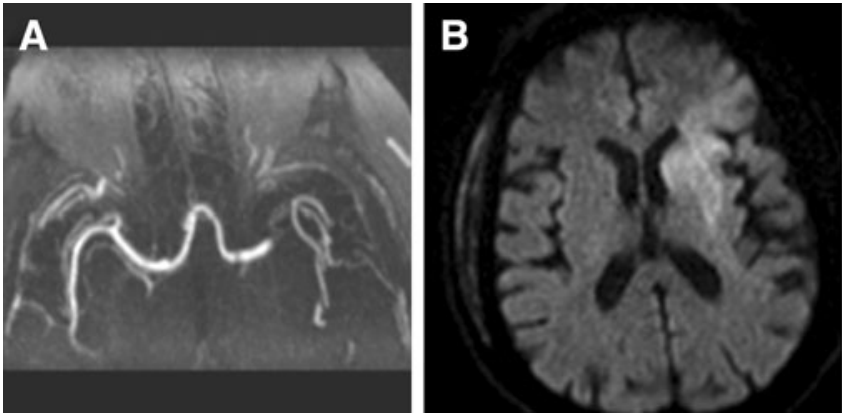

Fig. 3 A 63-year-old man with a history of atrial fibrillation presented with acute onset of right sided weakness, global aphasia, and right visual field deficit. a MR angiography (MRA) revealed occlusion of the proximal left middle cerebral artery (MCA). b Diffusion-weighted imaging (DWI) showed an area of restricted diffusion involving the caudate, insula, and portion of the left frontal lobe. $\mathbf{c}$ MR perfusion showed elevated time to peak in a large portion of the MCA territory, and d FLAIR imaging revealed hyperintense

MR angiography (MRA) is another commonly used technique to evaluate structural collaterals. Besides structural information on flow-limiting lesions, MRI techniques provide additional information on cerebrovascular hemodynamics. Information on cerebral blood flow can be obtained in a quantitative manner using a technique known as NOVA [19] and has been used to demonstrate collateral flow from the posterior to anterior circulation in the setting of anterior circulation stenosis. In addition, FLAIR vascular hyperintensity likely represents disordered or disorganized flow in the setting of arterial disease and is another means of indirectly assessing collateral flow [20-24]. This finding can be seen in acute ischemic stroke, as shown in Fig. 3, intracranial stenosis, TIA, or moyamoya syndrome. Unlike other forms of static vessel imaging, it implies a functional deficit and serves as a marker of leptomeningeal collateral perfusion to tissue at risk. The finding is highly specific and sensitive in the setting of arterial occlusion $[23,24]$.
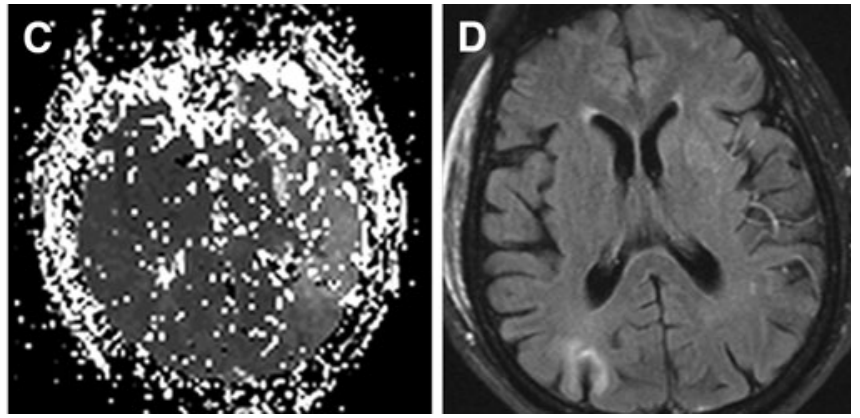

vasculature consistent with collateral flow that likely led to preservation of a large portion of the posterior MCA territory. Given the evidence of preserved collateral flow, in spite of being outside the window for intravenous tissue plasminogen activator, the patient was enrolled in a randomized trial comparing the thrombolytic desmoteplase versus placebo (DIAS IV) [67]. Two days later, he had nearly full strength on his right side and had regained his vision and much of his language ability

\section{Functional Collateral Imaging Defines Territory at Risk}

Beyond structural assessments of collateral circulation, advances in perfusion-based imaging have allowed for functional evaluations of the quality of collateral blood flow. These studies are of particular importance in both acute and chronic arterial occlusive disorders as they provide crucial information on the health of the underlying brain parenchyma. Regardless of the modality employed, a finding of hypoperfusion indicates a territory at risk, and hyperperfusion, in the setting of poor collaterals, may represent the potential risk of hemorrhagic transformation through increased permeability and blood-brain barrier disruption [25].

CT perfusion techniques are becoming increasingly widespread in the evaluation of acute ischemic stroke, an example of which is given in Fig. 4. This modality offers advantages of wide availability, speed, and the opportunity for quantitative measurements. Reperfusion, as defined in 


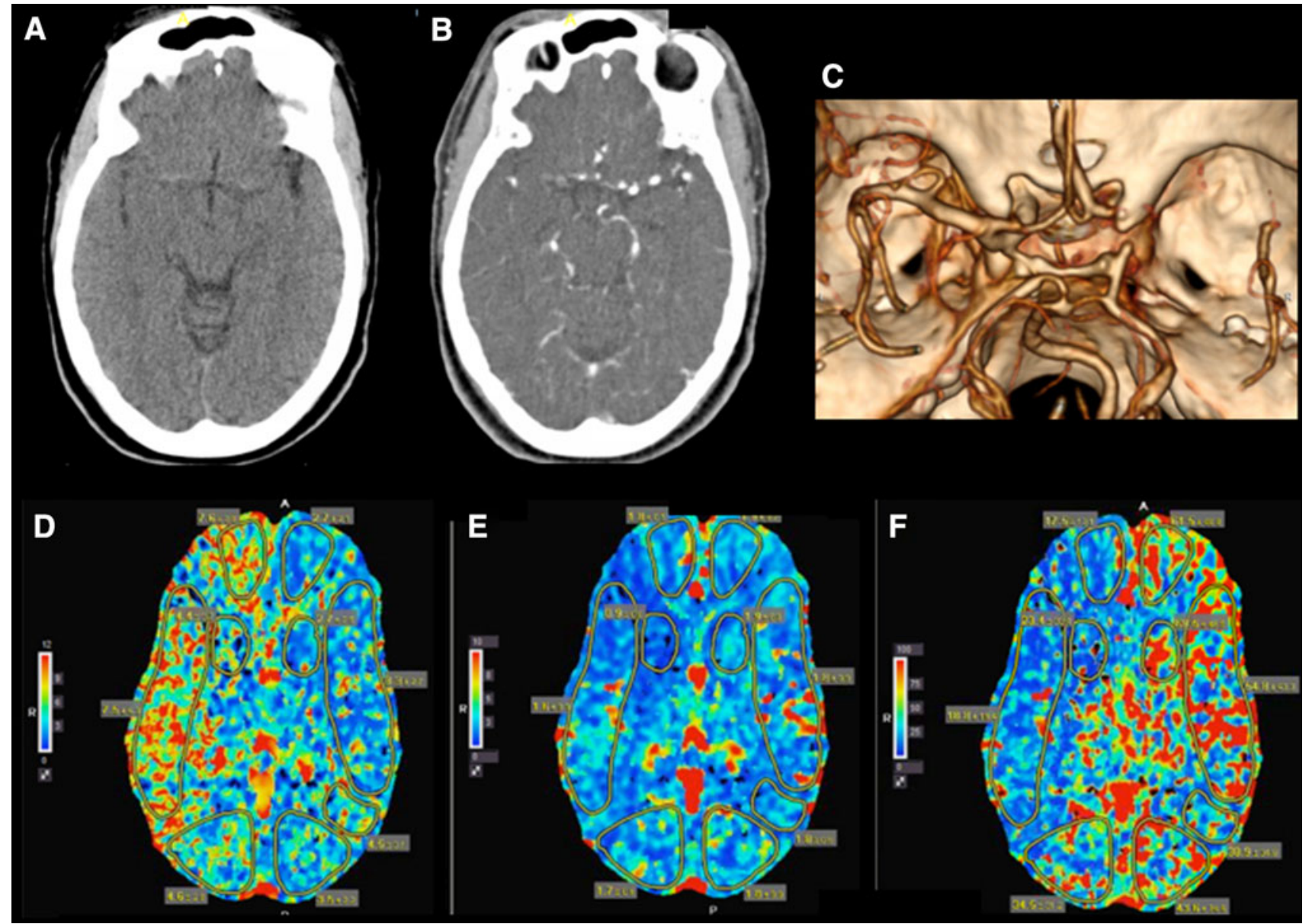

Fig. 4 A 62-year-old woman with a history of hypertension and hyperlipidemia presented with acute-onset left-sided weakness and loss of consciousness. a Non-contrast head CT revealed possible thrombus in the right middle cerebral artery (MCA) and blurring of the gray-white junction of the right insula. b CT angiography (CTA) showed occlusion of the right MCA and internal carotid artery (ICA), also seen on c reformatted CTA images. CT perfusion revealed d elevated mean transit time in the right MCA territory with

CT perfusion terms of decreased mean transit time, has been shown to correlate with final infarct volume more accurately than recanalization [26]. Operative experience, however, is crucial in the interpretation of these images. Beyond the importance of appropriately selected arterial and venous input functions, there are a number of scenarios that may result in false-negative or -positive findings. These instances include anatomic variability, such as arterial stenosis, which may lead to overestimation of the size of the ischemic penumbra; as a result, CT perfusion must be interpreted with concurrent CTA imaging. Importantly, clinical entities that can mimic acute ischemic stroke can also result in identical $\mathrm{CT}$ perfusion findings. Focal seizure may lead to the finding of ipsilateral hyperperfusion with diminished mean transit time and elevated $\mathrm{CBF}$, but may be interpreted as contralateral hyperperfusion [27, 28]. Conversely, hemiplegic diminished e cerebral blood volume and $\mathbf{f}$ cerebral blood flow in the anterior more than posterior portion of the MCA territory. The portion of parenchyma with elevated mean transit time and relatively preserved cerebral blood volume was felt to be tissue sustained by collateral flow though at risk of infarction over time. The patient was treated with intravenous thrombolysis as well as embolectomy and had recovered spontaneous movement in the left side of her body the next day

migraine, another disorder that can result in acute neurological changes easily conflated with ischemic stroke, may result in a finding of focal hypoperfusion identical to that of stroke. Without the diffusion-weighted imaging available with MRI, the distinction between the two entities based on imaging would be impossible [29]. This confluence of clinical and imaging mimicry compounded with the inability to definitively visualize acute ischemic lesions with CTbased parenchymal evaluations may limit the utility of CT perfusion as a stand-alone metric. As a result, the clinical assessment of the patient through history and physical exam remains of particular importance in the setting of CT-based perfusion acute stroke protocols [30].

MR-based perfusion is also widely used in the assessment of ischemic brain disease. This study is most commonly performed using a bolus of contrast and assessing 
flow dynamics by monitoring the passage of the bolus through the vessels and parenchyma. A more recent technique known as arterial spin labeling (ASL) magnetically "labels" arterial blood water using radiofrequency pulses that then decay with $\mathrm{T} 1$ relaxation and allows for quantitative assessments of regional cerebral blood flow without the need for a tracer. This technique has been used in moyamoya disease [31], acute ischemic stroke [32], and chronic arterial stenoses [33]. Compared to dynamic susceptibility contrast-enhanced perfusion MRI, ASL may demonstrate hyperperfusion more overtly [32].

Transcranial Doppler (TCD) can provide physiological information on the status of cerebral collaterals. Changes in flow directionality (i.e., in the ophthalmic artery or anterior communicating artery) can inform compensatory collateral changes. Similarly, flow diversion, defined as increased velocity in vessels ipsilateral to a stenosis or occlusion as compared to contralateral controls, has been shown to correlate with the presence of leptomeningeal collaterals [34].

Other methods of measuring cerebral perfusion include PET and nuclear medicine approaches such as SPECT. These techniques, in addition to the ones mentioned above, can be combined with a vasodilatory stimulus to determine the resilience of the cerebral circulation to ischemic insults, termed the cerebrovascular reserve (CVR). This measurement takes into account all the compensatory changes available to the brain to preserve cerebral blood flow in the setting of acute ischemia. Intravenous acetazolamide and inhaled $\mathrm{CO}_{2}$ are the most common vasodilatory stimuli, and an inability to recruit additional blood flow in response to their administration signifies impaired collateral flow and diminished CVR.

\section{Collateral Grading Scales}

A multitude of grading scales have been developed to describe the presence and quality of collaterals based on CT, MRI, TCD, and angiographic evaluations. These scales involve both structural and functional aspects of collateral flow. There is, however, significant inconsistency in how the grading and scaling is performed as well as the interrater reliability. For angiographic assessments, the ASITN/ SIR scales [35] remain the most commonly used. This lack of consensus may contribute to an overall under appreciation of the fundamental role of collateral circulation in outcomes following acute ischemic stroke.

\section{Clinical Impact}

\section{Chronic Arterial Occlusive Disease}

Assessments of both structural and functional aspects of collateral circulation are most commonly used in determinations of the risk of ischemia from arterial occlusive disorders. Carotid stenosis in particular is one of the most well-studied applications. An estimated $15-20 \%$ of ischemic strokes have been attributed to ipsilateral carotid atherosclerotic disease [36], and while the degree of luminal narrowing is the measurement most commonly used to define hemodynamically significant stenosis, this measurement does not take into account the downstream hemodynamic changes. Such assessments may be particularly important in the case of asymptomatic carotid disease, in which case the utility of revascularization procedures is less clear, as the reduction in stroke risk may equal the surgical risk [37].

Several studies have evaluated the impairment in functional collateral flow in the setting of chronic stenosis or occlusion of the internal carotid or middle cerebral artery, and attempted to correlate those changes with risk of subsequent stroke or TIA [38-44]. A recent meta-analysis found a significant odds ratio of 4 between impairment in CVR and subsequent risk of ischemic disease in both symptomatic and asymptomatic carotid disease [45].

Similar approaches have been used to assess the risk of downstream ischemia in the setting of intracranial stenosis, one the most common causes of strokes worldwide [4]. Treatment of symptomatic lesions with angioplasty and stenting has been shown to improve CVR [46, 47]. A recent trial of endovascular stenting for these patients, however, failed to demonstrate a reduction in the risk of stroke likely because of periprocedural complications [4]. Even in the setting of demonstrable hypoperfusion using the techniques listed above, the optimal management strategy for this disorder remains unclear.

Moyamoya syndrome or disease is a scenario in which assessments of collateral flow play a pivotal role in clinical decision-making. This disorder is a potentially, progressive obliterative vasculopathy in which the normally large-caliber proximal vessels of the brain are obstructed and replaced by thin, ineffective perforating vascular channels [48]. In a sense, moyamoya syndrome represents the most extreme example of collateral development and one of the greatest challenges to the secondary sources of blood supply to the brain. Patients with this disorder may come to medical attention as a result of symptoms associated with chronic cerebral hypoperfusion, such as headaches, ischemic TIA/stroke, or cognitive dysfunction. As in the case depicted in Fig. 1, perfusion studies are routinely used in patient selection for evaluation for revascularization and in follow-up assessments of efficacy $[49,50]$. Functional collateral imaging has demonstrated a link between frontal lobe hypoperfusion and frontally mediated cognitive dysfunction, and, importantly, improvement in both parameters with revascularization [51, 52]. 


\section{Acute Arterial Occlusive Disorders}

Acute ischemic stroke is one of the most promising areas for real-time assessments of collateral flow and is also one of the areas with the greatest need for these techniques. Patient selection for acute revascularization procedures remains highly controversial, particularly in the case of endovascular thrombectomy $[1 \bullet \bullet, 2]$. The initial efforts in this field using intravenous thrombolysis did not include an evaluation of cerebral hemodynamics and instead used time from last known well as a proxy [53]. As a result, the existing metrics to determine patient eligibility for intravenous thrombolysis, as well as endovascular reperfusion in many cases, are based on time alone [54]. This approach, of course, does not take into consideration the significant variability in hemodynamic response due to collateral circulation anatomy. Without taking this factor into account, we may be undertreating patients with a robust collateral circulation who may be suitable for revascularization many hours after symptom onset and over-treating those with a meager response who may have already suffered infarction of the entire vascular territory well before the arbitrarily defined time point. More recent studies have begun to address this issue and found that patients with poor collaterals have been shown to present to the hospital earlier and have poorer outcomes from acute stroke. On the other hand, the ability to recruit collateral vessels has been shown to be timedependent and correlate with stabilization of clinical symptoms [55]. A recent assessment of intravenous thrombolysis moved to incorporate collateral assessments into the decision and found that in patients who are able to maintain perfusion to a suitable level in spite of an acute arterial occlusions, treatment at up to $6 \mathrm{~h}$ may be safe [56].

The presence of collaterals has been shown to have an impact on the ultimate injury associated with acute arterial occlusions. Regional assessments of collateral networks can predict the ultimate infarct area in the setting of comparable sites of occlusion [57]. A favorable vascular profile consisting of an intact circle of Willis and MAP was shown to predict improved outcome after stroke [58]. Diminished collateral scores based on CTA have been shown to correlate with larger territories of ischemia in the setting of occlusions of the internal carotid or middle cerebral artery $[55,59]$. Conversely, the presence of CTAdefined leptomeningeal collaterals has also been shown to correlate with improved functional outcome in acute ischemic stroke $[60,61]$. In addition, the finding of very low cerebral blood volume on perfusion imaging may predict hemorrhage after thrombolytic therapy even more than the volume of diffusion-weighted imaging positivity [62].

Patient selection for intra-arterial revascularization techniques also benefits from inclusion criteria that take into account cerebral perfusion [63]. In patients undergoing endovascular revascularization therapy, higher collateral grades have been demonstrated to lead to improved recanalization rates; similarly, patients with poor collaterals were shown to not benefit even with successful recanalization of the occlusive lesion [64]. Patients with poor collaterals tend to present with higher volumes of perfusion/diffusion mismatch versus areas of benign hypoperfusion compared to patients with robust collaterals, who had larger areas of benign hypoperfusion. Similarly, after recanalization therapy, patients with poor collaterals were more likely to suffer expansion of the infarction area in the region of impaired perfusion [65]. After endovascular reperfusion, collateral status predicts hemorrhage rate as well [64].

\section{Conclusion}

The presence and quality of collateral circulation has become a fundamental feature in the evaluation and treatment of cerebral ischemia. Though recognized for hundreds of years, the beneficial influence of collateral flow has now gained significant attention due to widely available, rapid, and real-time non-invasive imaging techniques [66]. Collateral imaging has become a cornerstone in the evaluation of patients with acute and chronic cerebral ischemia by informing the diagnosis, treatment, and follow-up for these patients. These modalities provide rich detail on the quality and quantity of cerebral blood flow, which in the end is the sole determinant of tissue viability.

\section{Compliance with Ethics Guidelines}

Conflict of Interest Sunil A. Sheth declares no conflict of interest. David S. Liebeskind's institution receives funding for his consultancy for Covidien and Stryker. His institution also receives funding through NIH/NINDS research grants.

Human and Animal Rights and Informed Consent This article does not contain any studies with human or animal subjects performed by the authors.

\section{References}

Papers of particular interest, published recently, have been highlighted as:

- Of importance

- Of major importance

1. • Kidwell CS, Jahan R, Gornbein J, Alger JR, Nenov V, Ajani Z, et al. A trial of imaging selection and endovascular treatment for ischemic stroke. N Engl J Med 2013;368:914-23. This pivotal 
study, known as MR RESCUE, was the first to use perfusion imaging in a large, randomized trial setting evaluating endovascular therapy for acute ischemic stroke. Though the study did not base treatment decisions on the findings of perfusion imaging, future trials will likely incorporate the imaging methodology established within this trial.

2. Broderick JP, Palesch YY, Demchuk AM, Yeatts SD, Khatri P, Hill MD, et al. Endovascular therapy after intravenous t-PA versus t-PA alone for stroke. N Engl J Med. 2013;368:893-903.

3. - Powers WJ, Clarke WR, Grubb RL, Videen TO, Adams HP, Derdeyn CP, et al. Extracranial-intracranial bypass surgery for stroke prevention in hemodynamic cerebral ischemia: the Carotid Occlusion Surgery Study randomized trial. JAMA. 2011;306:1983-92. This trial, abbreviated COSS, evaluated patients with evidence of hemodynamic changes on PET imaging from carotid occlusions. Though the surgical intervention studied in the trial failed to show benefit, the approach of perfusion imaging to identify patients meriting additional treatment is likely to be continued.

4. Chimowitz MI, Lynn MJ, Derdeyn CP, Turan TN, Fiorella D, Lane BF, et al. Stenting versus aggressive medical therapy for intracranial arterial stenosis. N Engl J Med. 2011;365:993-1003.

5. Liebeskind DS. Collateral Circulation. Stroke. 2003;34:2279-84.

6. Powers WJ. Cerebral hemodynamics in ischemic cerebrovascular disease. Ann Neurol. 1991;29:231-40.

7. Lippert H, Pabst R. Arterial variations in man: classification. Munich: JF Bergmann Verlag; 1985.

8. Zanette EM, Fieschi C, Bozzao L, Roberti C, Toni D, Argentino $\mathrm{C}$, et al. Comparison of cerebral angiography and transcranial Doppler sonography in acute stroke. Stroke. 1989;20:899-903.

9. Gillilan LA. Potential collateral circulation to the human cerebral cortex. Neurology. 1974;24:941-8.

10. Chen J, Chopp M. Angiogenesis and arteriogenesis as stroke targets. Translational stroke research. New York: Springer; 2012. p. 231-49.

11. Dor Y, Keshet E. Ischemia-driven angiogenesis. Trends Cardiovasc Med. 1997;7:289-94.

12. van Royen N, Piek JJ, Buschmann I, Hoefer I, Voskuil M, Schaper W. Stimulation of arteriogenesis; a new concept for the treatment of arterial occlusive disease. Cardiovasc Res. 2001;49:543-53.

13. Coyle P, Heistad DD. Development of collaterals in the cerebral circulation. J Vasc Res. 1991;28:183-9.

14. Toyoda K, Minematsu K, Yamaguchi T. Long-term changes in cerebral blood flow according to different types of ischemic stroke. J Neurol Sci. 1994;121:222-8.

15. Faber JE, Zhang H, Lassance-Soares RM, Prabhakar P, Najafi $\mathrm{AH}$, Burnett MS, et al. Aging causes collateral rarefaction and increased severity of ischemic injury in multiple tissues. Arterioscler Thromb Vasc Biol. 2011;31:1748-56.

16. Shuaib A, Butcher K, Mohammad AA, Saqqur M, Liebeskind DS. Collateral blood vessels in acute ischaemic stroke: a potential therapeutic target. Lancet Neurol. 2011;10:909-21.

17. Willems PWA, Taeshineetanakul P, Schenk B, Brouwer PA, Terbrugge KG, Krings T. The use of 4D-CTA in the diagnostic work-up of brain arteriovenous malformations. Neuroradiology. 2012;54:123-31.

18. Frölich AMJ, Schrader D, Klotz E, Schramm R, Wasser K, Knauth M, et al. 4D CT angiography more closely defines intracranial thrombus burden than single-phase CT angiography. Am J Neuroradiol. 2013;34:1908-13.

19. Ruland S, Ahmed A, Thomas K, Zhao M, Amin-Hanjani S, Du X, et al. Leptomeningeal collateral volume flow assessed by quantitative magnetic resonance angiography in large-vessel cerebrovascular disease. J Neuroimaging. 2009;19:27-30.

20. Kamran S, Bates V, Bakshi R, Wright P, Kinkel W, Miletich R. Significance of hyperintense vessels on FLAIR MRI in acute stroke. Neurology. 2000;55:265-9.
21. Lee KY, Latour LL, Luby M, Hsia AW, Merino JG. Distal hyperintense vessels on FLAIR An MRI marker for collateral circulation in acute stroke? Neurology. 2009;72:1134-9.

22. Sanossian N, Saver JL, Alger JR, Kim D, Duckwiler GR, Jahan $\mathrm{R}$, et al. Angiography reveals that fluid-attenuated inversion recovery vascular hyperintensities are due to slow flow, not thrombus. Am J Neuroradiol. 2009;30:564-8.

23. Schellinger PD, Chalela JA, Kang D-W, Latour LL, Warach S. Diagnostic and prognostic value of early MR Imaging vessel signs in hyperacute stroke patients imaged. AJNR. $2005 ; 26: 618-24$.

24. Azizyan A, Sanossian N, Mogensen MA, Liebeskind DS. Fluidattenuated inversion recovery vascular hyperintensities: an important imaging marker for cerebrovascular disease. Am J Neuroradiol. 2011;32:1771-5.

25. Kidwell CS, Saver JL, Mattiello J, Starkman S, Viñuela F, Duckwiler G, et al. Diffusion-perfusion MRI characterization of post-recanalization hyperperfusion in humans. Neurology. 2001;57:2015-21.

26. Soares BP, Tong E, Hom J, Cheng S-C, Bredno J, Boussel L, et al. Reperfusion is a more accurate predictor of follow-up infarct volume than recanalization: a proof of concept using CT in acute ischemic stroke patients. Stroke. 2010;41:e34-40.

27. Masterson K, Vargas MI, Delavelle J. Postictal deficit mimicking stroke: role of perfusion CT. J Neuroradiol. 2009;36:48-51.

28. Hauf M, Slotboom J, Nirkko A, von Bredow F, Ozdoba C, Wiest $\mathrm{R}$. Cortical regional hyperperfusion in nonconvulsive status epilepticus measured by dynamic brain perfusion CT. Am J Neuroradiol. 2009;30:693-8.

29. Hansen JM, Schytz HW, Larsen VA, Iversen HK, Ashina M. Hemiplegic migraine aura begins with cerebral hypoperfusion: imaging in the acute phase. Headache. 2011;51:1289-96.

30. Lui YW, Tang ER, Allmendinger AM, Spektor V. Evaluation of CT perfusion in the setting of cerebral ischemia: patterns and pitfalls. Am J Neuroradiol. 2010;31:1552-63.

31. Zaharchuk G, Do HM, Marks MP, Rosenberg J, Moseley ME, Steinberg GK. Arterial spin-labeling MRI can identify the presence and intensity of collateral perfusion in patients with moyamoya disease. Stroke. 2011;42:2485-91.

32. Wang DJJ, Alger JR, Qiao JX, Hao Q, Hou S, Fiaz R, et al. Better late than never: the long journey for noncontrast arterial spin labeling perfusion imaging in acute stroke. Stroke. 2012;43:931-2.

33. Kamano H, Yoshiura T, Hiwatashi A, Abe K, Togao O, Yamashita $\mathrm{K}$, et al. Arterial spin labeling in patients with chronic cerebral artery steno-occlusive disease: correlation with (15)OPET. Acta Radiol. 2013;54:99-106.

34. Kim Y, Sin D-S, Park H-Y, Park M-S, Cho K-H. Relationship between flow diversion on transcranial Doppler sonography and leptomeningeal collateral circulation in patients with middle cerebral artery occlusive disorder. J Neuroimaging. 2009;19: 23-6.

35. Higashida RT, Furlan AJ, Roberts H, Tomsick T, Connors B, Barr J, et al. Trial design and reporting standards for intra-arterial cerebral thrombolysis for acute ischemic stroke. Stroke. 2003;34:e109-37.

36. Chaturvedi S, Bruno A, Feasby T, Holloway R, Benavente O, Cohen SN, et al. Carotid endarterectomy-an evidence-based review: report of the Therapeutics and Technology Assessment Subcommittee of the American Academy of Neurology. Neurology. 2005;65:794-801.

37. Halliday A, Mansfield A, Marro J, Peto C, Peto R, Potter J, et al. Prevention of disabling and fatal strokes by successful carotid endarterectomy in patients without recent neurological symptoms: randomised controlled trial. Lancet. 2004;363:1491-502.

38. King A, Serena J, Bornstein NM, Markus HS. ACES Investigators. Does impaired cerebrovascular reactivity predict stroke risk 
in asymptomatic carotid stenosis? A prospective substudy of the asymptomatic carotid emboli study. Stroke. 2011;42:1550-5.

39. Isozaki M, Arai Y, Kudo T, Kiyono Y, Kobayashi M, Kubota T, et al. Clinical implication and prognosis of normal baseline cerebral blood flow with impaired vascular reserve in patients with major cerebral artery occlusive disease. Ann Nucl Med. 2010;24:371-7.

40. Kimiagar I, Bass A, Rabey JM, Bornstein NM, Gur AY. Longterm follow-up of patients with asymptomatic occlusion of the internal carotid artery with good and impaired cerebral vasomotor reactivity. Eur J Neurol. 2010;17:1285-90.

41. Kuroda S, Houkin K, Kamiyama H, Mitsumori K, Iwasaki Y, Abe H. Long-term prognosis of medically treated patients with internal carotid or middle cerebral artery occlusion: can acetazolamide test predict it? Stroke. 2001;32:2110-6.

42. Markus HS. Reduced cerebral blood flow in white matter in ischaemic leukoaraiosis demonstrated using quantitative exogenous contrast based perfusion MRI. J Neurol Neurosurg Psychiatry. 2000;69:48-53.

43. Ogasawara K, Ogawa A, Terasaki K, Shimizu H, Tominaga T, Yoshimoto T. Use of cerebrovascular reactivity in patients with symptomatic major cerebral artery occlusion to predict 5-year outcome: comparison of xenon-133 and iodine-123-IMP singlephoton emission computed tomography. J Cereb Blood Flow Metab. 2002;22:1142-8.

44. Reinhard M, Gerds TA, Grabiak D, Zimmermann PR, Roth M, Guschlbauer B, et al. Cerebral dysautoregulation and the risk of ischemic events in occlusive carotid artery disease. J Neurol. 2008;255:1182-9.

45. Gupta A, Chazen JL, Hartman M, Delgado D, Anumula N, Shao $\mathrm{H}$, et al. Cerebrovascular reserve and stroke risk in patients with carotid stenosis or occlusion: a systematic review and metaanalysis. Stroke. 2012;43:2884-91.

46. Attyé A, Villien M, Tahon F, Warnking J, Detante O, Krainik A. Normalization of cerebral vasoreactivity using BOLD MRI after intravascular stenting. Hum Brain Mapp. 2013;. doi:10.1002/ hbm. 22255 .

47. Abe AA, Ueda TT, Ueda MM, Nogoshi SS, Nishiyama YY, Katayama YY. Symptomatic middle cerebral artery stenosis treated by percutaneous transluminal angioplasty: improvement of cerebrovascular reserves. Interv Neuroradiol. 2012;18:213-20.

48. Liu X, Zhang D, Shuo W, Zhao Y, Wang R, Zhao J. Long term outcome after conservative and surgical treatment of haemorrhagic moyamoya disease. J Neurol Neurosurg Psychiatry. 2013;84:258-65.

49. Andaluz N, Choutka O, Vagal A, Strunk R, Zuccarello M. Patient selection for revascularization procedures in adult moyamoya disease based on dynamic perfusion computerized tomography with acetazolamide challenge (PCTA). Neurosurg Rev. 2010;33:225-33.

50. Schubert GA, Weinmann C, Seiz M, Gerigk L, Weiss C, Horn P, et al. Cerebrovascular insufficiency as the criterion for revascularization procedures in selected patients: a correlation study of xenon contrast-enhanced CT and PWI. Neurosurg Rev. 2009;32:29-35 discussion35-6.

51. Calviere L, Catalaa I, Marlats F, Viguier A, Bonneville F, Cognard $\mathrm{C}$, et al. Correlation between cognitive impairment and cerebral hemodynamic disturbances on perfusion magnetic resonance imaging in European adults with moyamoya disease. J Neurosurg. 2010;113:753-9.
52. Song YS, Oh SW, Kim YK, Kim S-K, Wang K-C, Lee DS. Hemodynamic improvement of anterior cerebral artery territory perfusion induced by bifrontal encephalo(periosteal)synangiosis in pediatric patients with moyamoya disease: a study with brain perfusion SPECT. Ann Nucl Med. 2011;26:47-57.

53. Tissue plasminogen activator for acute ischemic stroke. The National Institute of Neurological Disorders and Stroke rt-PA Stroke Study Group. N Engl J Med. 1995;333:1581-7.

54. Tissue plasminogen activator for acute ischemic stroke. The National Institute of Neurological Disorders and Stroke rt-PA Stroke Study Group. N Engl J Med. 1995;333:1581-7.

55. Maas MB, Lev MH, Ay H, Singhal AB, Greer DM, Smith WS, et al. Collateral vessels on CT angiography predict outcome in acute ischemic stroke. Stroke. 2009;40:3001-5.

56. Albers GW, Thijs VN, Wechsler L, Kemp S, Schlaug G, Skalabrin $\mathrm{E}$, et al. Magnetic resonance imaging profiles predict clinical response to early reperfusion: the diffusion and perfusion imaging evaluation for understanding stroke evolution (DEFUSE) study. Ann Neurol. 2006;60:508-17.

57. Cheng B, Golsari A, Fiehler J, Rosenkranz M, Gerloff C, Thomalla G. Dynamics of regional distribution of ischemic lesions in middle cerebral artery trunk occlusion relates to collateral circulation. J Cereb Blood Flow Metab. 2010;31:36-40.

58. Schellinger PD, Köhrmann M, Liu S, Dillon WP, Nogueira RG, Shuaib A, et al. Favorable vascular profile is an independent predictor of outcome: a post hoc analysis of the safety and efficacy of NeuroFlo Technology in Ischemic Stroke trial. Stroke. 2013;44:1606-8.

59. Souza LCS, Yoo AJ, Chaudhry ZA, Payabvash S, Kemmling A, Schaefer PW, et al. Malignant CTA collateral profile is highly specific for large admission DWI infarct core and poor outcome in acute stroke. Am J Neuroradiol. 2012;33:1331-6.

60. Lima FO, Furie KL, Silva GS, Lev MH, Camargo ECS, Singhal $\mathrm{AB}$, et al. The pattern of leptomeningeal collaterals on ct angiography is a strong predictor of long-term functional outcome in stroke patients with large vessel intracranial occlusion. Stroke. 2010;41:2316-22.

61. Tan IYL, Demchuk AM, Hopyan J, Zhang L, Gladstone D, Wong $\mathrm{K}$, et al. CT angiography clot burden score and collateral score: correlation with clinical and radiologic outcomes in acute middle cerebral artery infarct. AJNR. 2009;30:525-31.

62. Campbell BCV, Christensen S, Butcher KS, Gordon I, Parsons MW, Desmond PM, et al. Regional very low cerebral blood volume predicts hemorrhagic transformation better than diffusion-weighted imaging volume and thresholded apparent diffusion coefficient in acute ischemic stroke. Stroke. 2009;41:82-8.

63. Lansberg MG, Straka M, Kemp S, Mlynash M, Wechsler LR, Jovin TG, et al. MRI profile and response to endovascular reperfusion after stroke (DEFUSE 2): a prospective cohort study. Lancet Neurol. 2012;11:860-7.

64. Bang OY, Saver JL, Kim SJ, Kim GM, Chung CS, Ovbiagele B, et al. Collateral flow predicts response to endovascular therapy for acute ischemic stroke. Stroke. 2011;42:693-9.

65. Bang OY, Saver JL, Buck BH, Alger JR, Starkman S, Ovbiagele $\mathrm{B}$, et al. Impact of collateral flow on tissue fate in acute ischaemic stroke. J Neurol Neurosurg Psychiatry. 2008;79:625-9.

66. Liebeskind DS. The currency of collateralcirculation in acute ischemic stroke. Nat Rev Neurol. 2009;5:645-6.

67. http://clinicaltrials.gov/ct2/show/NCT00856661. Accessed 5 Nov 2013. 